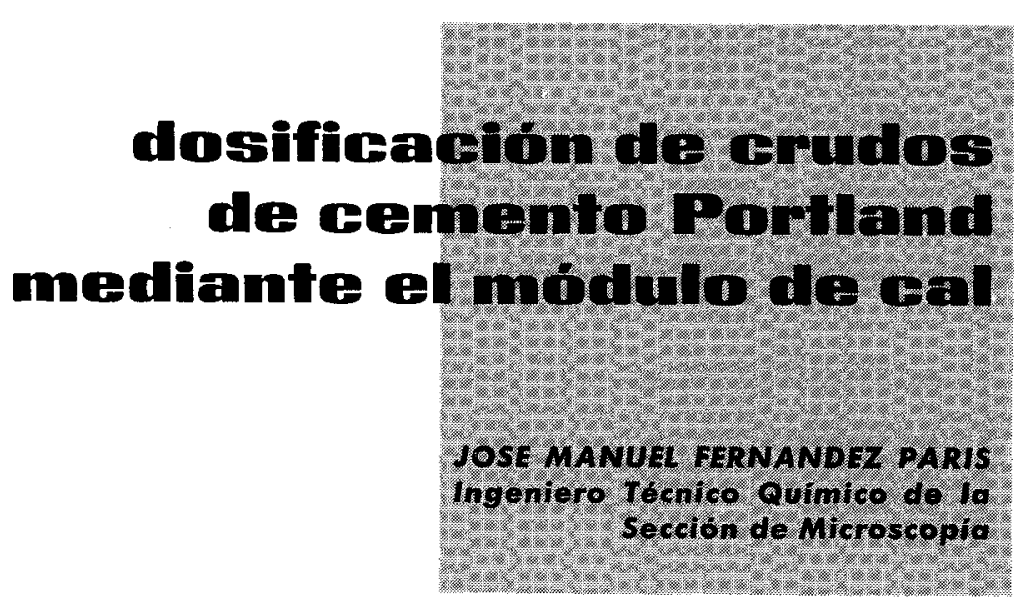

El crudo de un cemento portland es una mezcla artificial de materiales, convenientemente dosificada, que, por ulterior molienda y cocción, origina el clínker.

Las materias primas principales están integradas por el grupo de las calizas y las arcillas, así como por el intermedio de las margas. Las calizas son aportadoras del $\mathrm{CaO}$, denominado cal u óxido de cal, único óxido, de los cuatro principales ( $\mathrm{SiO}_{2}, \mathrm{Al}_{2} \mathrm{O}_{3}$, $\mathrm{Fe}_{2} \mathrm{O}_{3}$ y CaO), que presenta carácter básico. Las margas, como rocas de composición intermedia entre las arcillas y las calizas, aportan, en cantidad variable, la cal y los componentes ácidos.

Además de las citadas existen las materias primas auxiliares, también denominadas de corrección, cuya misión es suministrar uno o varios de los componentes que puedan faltar en las materias primas. Como corrector de sílice se pueden citar las arenas; de alúmina o de alúmina y óxido férrico, las bauxitas; de óxido férrico, las piritas; de silice y alúmina conjuntamente, las cenizas y puzolanas.

Desde los comienzos se trató de establecer relaciones entre los componentes principales, con el fin de destacar alguna función peculiar que éstos desempeñan en el conglomerante. Estas relaciones reciben la denominación de Indices y Módulos. Existe una gran diversidad de éstos, cuya exposición razonada ocuparía mucho espacio y posiblemente desvirtuaria determinados conceptos.

Los más importantes, en cuanto a fabricación y clasificación de cementos, son: Módulo hidráulico:

$$
\mathrm{MH}=\frac{\mathrm{CaO}}{\mathrm{SiO}_{2}+\overline{\mathrm{Al}}_{2} \mathrm{O}_{3}+\overline{\mathrm{Fe}}_{2} \overline{\mathrm{O}}_{3}} .
$$

Su finalidad es poder evaluar las características de basicidad de un cemento, mediante una relación entre el óxido de cal $(\mathrm{CaO})$ y la suma de los componentes ácidos $\left(\mathrm{SiO}_{2}+\mathrm{Al}_{2} \mathrm{O}_{3}+\mathrm{Fe}_{2} \mathrm{O}_{3}\right)$. 
En términos generales puede establecerse que al aumentar esta relación mejora la hidraulicidad del cemento. No obstante, esta expresión no facilita indicación cuantitativa del grado de combinación de la cal, puesto que los tres componentes que figuran en el denominador tienen distintos límites de saturación.

\begin{tabular}{|l|c|c|c|c|}
\hline \multicolumn{1}{|c|}{ CuAdro I } \\
\hline \multicolumn{1}{|c|}{ Denominación de los constituyentes } & \multicolumn{3}{|c|}{ Limite de Saturación } \\
\hline & $\frac{\mathrm{CaO} \%}{\mathrm{SiO} \%}$ & $\frac{\mathrm{CaO} \%}{\mathrm{Al}_{2} \mathrm{O}_{3} \%}$ & $\frac{\mathrm{CaO} \%}{\mathrm{Fe}_{2} \mathrm{O}_{3} \%}$ & $\mathrm{Al}_{2} \mathrm{O}_{3} \%$ \\
\hline $\begin{array}{l}\text { Silicato tricálcico, } \mathrm{SiO}_{2} \cdot 3 \mathrm{CaO}=\mathrm{SC}_{3} \\
\text { Silicato bicálcico, } \mathrm{SiO}_{2} \cdot 2 \mathrm{CaO}=\mathrm{SC}_{2}\end{array}$ & 2,80 & - & - & - \\
\hline $\begin{array}{l}\text { Aluminato tricálcico, } \mathrm{Al}_{2} \mathrm{O}_{3} \cdot 3 \mathrm{CaO}=\mathrm{AC}_{3} \\
\text { Ferrito aluminato tetracálcico, } \mathrm{Fe}_{2} \mathrm{O}_{3}, \mathrm{Al}_{2} \mathrm{O}_{3},\end{array}$ & $-1,87$ & - & - & - \\
\hline $4 \mathrm{CaO}=\mathrm{FAC}_{4}$ & - & 1,65 & - & - \\
\hline
\end{tabular}

\section{Módulo silícico}

La función del óxido silícico, $\mathrm{SiO}_{2}$, es originar los silicatos cálcicos, tanto tricálcico, $\mathrm{SC}_{3}$, como bicálcico, $\mathrm{SC}_{2}$, del clínker, compuestos ambos cristalinos que se forman merced a un conjunto de reacciones sólidas en el seno de un magma flúido integrado por aluminatos y ferrito aluminatos cálcicos, formados a partir del $\mathrm{Al}_{2} \mathrm{O}_{3}$ y $\mathrm{Fe}_{2} \mathrm{O}_{3}$. Por consiguiente, la función que desempeña el $\mathrm{SiO}_{2}$ es muy distinta de la del $\mathrm{Al}_{2} \mathrm{O}_{3}+$ $+\mathrm{Fe}_{2} \mathrm{O}_{3}$.

Es, por tanto, interesante conocer los valores indicados por la relación entre el $\mathrm{SiO}_{2}$ y la suma $\mathrm{Al}_{2} \mathrm{O}_{3}+\mathrm{Fe}_{2} \mathrm{O}_{3}$ :

$$
\mathrm{MS}=\frac{\mathrm{SiO}_{2}}{\mathrm{Al}_{2} \mathrm{O}_{3}+\overline{\mathrm{Fe}}_{2} \mathrm{O}_{3}} .
$$

Al aumentar el módulo silícico se increméntan los silicatos en el clínker, con la correspondiente disminución de los fundentes.

Los clínkeres de cemento portland suelen tener valores comprendidos entre 1,5 y 3 .

\section{Módulo de fundentes}

En éste se establece una relación entre los contenidos de alúmina $\left(\mathrm{Al}_{2} \mathrm{O}_{3}\right)$ y óxido férrico $\left(\mathrm{Fe}_{2} \mathrm{O}_{3}\right)$ :

$$
\mathrm{MF}=\frac{\mathrm{Al}_{2} \mathrm{O}_{3}}{\mathrm{Fe}_{2} \mathrm{O}_{3}}
$$

lo cual equivale a relacionar los componentes denominados fundentes y, por tanto, el aluminato tricálcico $\left(\mathrm{AC}_{3}\right)$ y el ferrito aluminato tetracálcico $\left(\mathrm{FAC}_{4}\right)$. 
En ausencia de $\mathrm{Fe}_{2} \mathrm{O}_{3}$, los valores de este módulo tienden a infinito, como sucede en Ios cementos blancos, con muy poco $\mathrm{FAC}_{4} \mathrm{y}$ un porcentaje elevado de $\mathrm{AC}_{3}$.

Cuando baja el contenido de $\mathrm{AC}_{3}$, los valores del módulo de fundentes van disminuyendo hasta el valor límite de 0,64 , con la desaparición total del $\mathrm{AC}_{3}$ y la sola presencia de $\mathrm{FAC}_{4}$ en la masa de fundentes. En este compuesto, $\mathrm{FAC}_{4}$, cada molécula de $\mathrm{Al}_{2} \mathrm{O}_{3}$ va unida a otra de $\mathrm{Fe}_{2} \mathrm{O}_{3}$ y, por ello, el módulo de fundentes será la relación entre los pesos moleculares de $\mathrm{Al}_{2} \mathrm{O}_{3}(102)$ y $\mathrm{Fe}_{2} \mathrm{O}_{3}(160)$ :

$$
\frac{\mathrm{Pm} \mathrm{Al}_{2} \mathrm{O}_{3}}{\mathrm{Pm} \mathrm{Fe}_{2} \mathrm{O}_{3}}=\frac{102}{160}=0,64 \text {. }
$$

Con un módulo de fundentes inferior a 0,64 no se consigue un clínker de cemento portland, sino que se entra en el campo də los cementos férricos, los cuales tienen un módulo próximo a $\mathbf{0 , 4 0}$.

Por consiguiente, el módulo de fundentes diferencia bastante bien los distintos tipos de cementos:

Módulo de fundentes próximo a infinito: cementos blancos.

Módulo de fundentes $3,00 \div 0,64:$ cementos portland normales.

Módulo de fundentes $0,64 \div 0,40$ : cementos férricos.

Indice de saturación en cal

Es la relación entre la cal contenida en el clinker y la cal máxima que puede ser fijada por los compuestos a su máxima basicidad, expresada en tanto por ciento:

$$
\mathrm{SC}=\frac{\mathrm{CaO} \text { total } \cdot 100}{\mathrm{CaO} \text { silicatos }+\mathrm{CaO} \text { aluminatos }+\mathrm{CaO} \text { ferritos }}
$$

Como el $\mathrm{FAC}_{4}$ puede expresarse $\mathrm{AC}_{3}+\mathrm{FC}$ y $\frac{\mathrm{CaO} \%}{\mathrm{Fe}_{2} \mathrm{O}_{3}^{\prime} \%}=0,35$, teniendo presente el cuadro I, se tiene:

$$
\mathrm{SC}=\frac{\mathrm{CaO} \cdot 100}{2,8 \mathrm{SiO}+1,65 \mathrm{~A}+0,35 \mathrm{~F}} .
$$

Si el índice de saturación en cal es menor que 100 , indica que la composición examinada puede soportar, al menos teóricamente, posteriores adiciones de cal; si es mayor que 100 habrá cal en exceso.

\section{Standard de cal}

Se ha demostrado que los cementos de tipo portland, independientemente de su módulo de fundentes, y en condiciones prácticas de cocción y enfriamiento, los componentes hidráulicos reaccionan siempre con la misma cantidad de cal, y ésta coincide exactamente con la fijada por Kühl mediante estudios estadísticos efectuados con gran número de cementos portland de buena calidad.

La cantidad máxima de cal que prácticamente se puede combinar, en condiciones de cocción y enfriamiento normales, recibe el nombre de cal standard y queda definida por:

$$
\text { Cal standard }=2,8 \mathrm{SiO}_{2}+1,1 \mathrm{~A}+0,7 \mathrm{~F} \text {. }
$$


Los coeficientes de dicha expresión corresponden a considerar que todo el silicato se halla como $\mathrm{SC}_{3}$, el aluminato como $\mathrm{AC}_{2}$, el ferrito como $\mathrm{FC}_{2}$.

Si en la expresión del índice de saturación de cal se sustituye el denominador por la cal standard, se obtiene una expresión que se denomina indice standard de saturación de cal.

$$
\mathrm{SC}=\frac{\mathrm{CaO} \cdot 100}{2,8 \mathrm{SiO}_{2}+1,1 \mathrm{Al}_{2} \mathrm{O}_{3}+\overline{0,7 \mathrm{Fe}_{2} \bar{O}_{3}}},
$$

que tiene una gran importancia, por que se ajusta a la realidad práctica. En dicha expresión el término $\mathrm{Fe}_{2} \mathrm{O}_{3}$ incluye el posible $\mathrm{Mn}_{2} \mathrm{O}_{3}$ y el $\mathrm{Al}_{2} \mathrm{O}_{3}$ el posible $\mathrm{TiO}_{2}$. La magnesia no se toma en consideración por quedar sin combinar.

El indice standard de saturación de cal puede definirse como la relación entre la cal total y la cal que puede ser fijada, desde una realidad práctica, por los compuestos, expresado en tanto por ciento.

En los cementos portland ordinarios el standard de cal suele oscilar entre 90 y 95 , mientras que en los de altas resistencias iniciales varía entre 95 y 98.

\section{Módulo de cal}

Ninguno de los módulos expuestos permite establecer una relación entre los dos silicatos que se forman en el clínker, o sea, entre el silicato tricálcico y el silicato bicálcico y que, como es sabido, tienen características bien distintas.

El silicato tricálcico tiene una hidratación y un endurecimiento muy rápido, así como un calor de hidratación elevado.

El silicato tricálcico tiene una hidratación y un endurecimiento lento $\mathrm{y}$, por tanto, un calor de hidratación bajo.

El módulo de cal

Utilizado sobre todo por la industria cementera italiana, ha sido perfectamente tratado por el profesor L. Santarelli (1). Su estructura es más compleja que la de los anteriormente definidos; pero presenta la enorme ventaja de establecer una estrecha unión entre los silicatos, constituyentes esenciales del clínker de cemento portland.

El módulo de cal puede definirse como la relación entre la cal, $\mathrm{CaO}$, que va a formar los silicatos y la sílice, $\mathrm{SiO}_{2}$, que con ella se combina.

Teniendo en cuenta el cuadro I y considerando que el $\mathrm{FAC}_{4}=\mathrm{AC}_{3}+\mathrm{FC}_{\text {: }}$

$$
\mathrm{MC}=\frac{\mathrm{CaO}-\left(1,65 \mathrm{Al}_{2} \mathrm{O}_{3}+0,35 \mathrm{Fe}_{2} \mathrm{O}_{3}\right)}{\mathrm{SiO}_{2}}
$$

Este módulo puede variar teóricamente en los cementos entre 1,87, que corresponde a la formación únicamente de $\mathrm{SC}_{2}: \frac{2 \mathrm{CaO}}{\mathrm{SiO}_{2}}=\frac{112}{60}=1,87$ y 2,80 en el caso de que 
sólo esté presente el $\mathrm{SC}_{3}$ :

$$
\mathrm{SC}_{3}: \frac{3 \mathrm{CaO}}{\mathrm{SiO}_{2}}=\frac{168}{60}=2,80 .
$$

En la práctica es imposible sobrepasar el valor de 2,60, por dificultades de técnica industrial en la cocción, e inclusive se hace difícil rebasar el valor de 2,40. Los buenos clínkeres tienen un valor de módulo de cal comprendido entre 2,45 y 2,55.

Si se tienen en cuenta las fórmulas dadas por Bogue para el Cálculo Potencial de Componentes :

$$
\begin{aligned}
\mathrm{SC}_{3} & =4,07 \mathrm{CaO}-7,60 \mathrm{SiO}_{2}-6,72 \mathrm{Al}_{2} \mathrm{O}_{3}-1,43 \mathrm{Fe}_{2} \mathrm{O}_{3}, \\
\mathrm{SC}_{2} & =2,87 \mathrm{SiO}_{2}-0,754 \mathrm{SC}_{3}, \\
\mathrm{AC}_{3} & =2,65 \mathrm{Al}_{2} \mathrm{O}_{3}-1,69 \mathrm{Fe}_{2} \mathrm{O}_{3}, \\
\mathrm{FAC}_{4} & =3,04 \mathrm{Fe}_{2} \mathrm{O}_{3} .
\end{aligned}
$$

Si se dividen los dos miembros de la expresión que da el valor del $\mathrm{SC}_{3}$ entre 4,07 , se tiene:

$$
\frac{\mathrm{SC}_{3}}{4,07}=\mathrm{CaO}-\left(1,65 \mathrm{Al}_{2} \mathrm{O}_{3}+0,35 \mathrm{Fe}_{2} \mathrm{O}_{3}\right)-1,87 \mathrm{SiO}_{2}
$$

Teniendo en cuenta que

$$
\mathrm{CaO}-\left(1,65 \mathrm{Al}_{2} \mathrm{O}_{3}+0,35 \mathrm{Fe}_{2} \mathrm{O}_{3}\right)=\text { Módulo de } \mathrm{Cal} \cdot \mathrm{SiO}_{2}
$$

y sustituyendo, tenemos:

$$
\begin{aligned}
\frac{\mathrm{SC}_{3}}{4,07} & =\mathrm{M} \cdot \mathrm{SiO}_{2}-1,87 \mathrm{SiO}_{2}, \\
\mathrm{SC}_{3} & =(4,07 \mathrm{M}-7,61) \mathrm{SiO}_{2} .
\end{aligned}
$$

La expresión que proporciona el valor del $\mathrm{SC}_{2}$ puede escribirse:

$$
\mathrm{SC}_{2}=2,87 \mathrm{SiO}_{2}-0,754 \mathrm{SiO}_{2}(4,07 \mathrm{M}-7,61),
$$

de donde:

$$
\mathrm{SC}_{2}=(8,61-3,07 \mathrm{M}) \mathrm{SiO}_{2},
$$

y, por tanto, el módulo de cal permite calcular la composición potencial de silicatos mediante las expresiones:

$$
\begin{array}{ll}
\mathrm{SC}_{2}=(8,61-3,07 \mathrm{M}) & \mathrm{SiO}_{2}, \\
\mathrm{SC}_{3}=(4,07 \mathrm{M}-7,61) & \mathrm{SiO}_{2} ;
\end{array}
$$

expresiones análogas a las propuestas por W. A. Kind y S. D. Okorokow (2) en función del grado de basicidad, (3), (4), (5) y (6). 


\section{Fundamentos de la dosificación de crudos}

El problema de la dosificación de crudos se resuelve siempre mediante sistemas de ecuaciones, que se establecen a partir de las composiciones de las materias primas principales y auxiliares de que se dispone.

Una mezcla se calificaría de unitaria si estuviera constituida por una sola materia prima, la cual, una vez calcinada, diera un producto que respondiese a la composición del clínker de cemento portland. Este hecho no acostumbra a darse en la realidad, y de ahi que las mezclas hayan de ser binarias (mezcla dosificada de dos materias primas), y ternarias (integradas por tres materiales). Se da, con muy poca frecuencia, el caso de tener que recurrir a mayor número; por lo tanto, puede considerarse que las mezclas cuaternarias se salen fuera de lo normal.

En los sistemas de ecuaciones que se plantean para el cálculo de una dosificación, siempre una de ellas expresa que la suma de los tantos por ciento de las materias primas elegidas para el crudo es igual a 100. Cada una de las ecuaciones restantes establece una condición (módulo hidráulico, módulo silícico, módulo de fundentes, grado de saturación, o standard de cal) impuesta al crudo.

Como en las dosificaciones binarias sólo se dispone de dos materias primas, únicamente se podrán establecer dos ecuaciones con dos incógnitas (cada una de las materias primas), y, puesto que una ecuación ha de expresar la suma a 100 de dichas materias, podrá imponerse sólo una condición al sistema.

De modo análogo en las dosificaciones ternarias, únicamente podrán ponerse dos condiciones; $y$ tres, a las cuaternarias.

Es preciso hacer notar también que es preferible que un crudo cumpla en primer lugar con índices o módulos en los cuales intervenga el mayor número posible de componentes y después con los de menor número. Así, es preferible que una mezcla binaria satisfaga antes un standard de cal, que al resto de los módulos, puesto que en aquél entran los cuatro óxidos mayores, mientras que en los módulos hidráulicos y silícicos intervienen tres, y sólo dos en el de fundentes.

El Dr. J. Calleja (7) ha tratado perfectamnte el tema, efectuando cálculos, incluso con mezclas cuaternarias, en función del standard cal, módulo hidráulico, módulo silícico y módulo de fundentes, así como dosificaciones basadas en la composición potencial.

Como el método que se va a exponer, basado en el módulo de cal, se ciñe en líneas generales al efectuado por el citado investigador, se explicará éste a grandes rasgos.

Consiste en determinar, para cada materia prima, el valor en que su composición difiere de las condiciones establecidas para el standard de cal y los restantes módulos.

Las diferencias que cada materia prima tiene respecto de los módulos impuestos se denominan con el término genérico de desviaciones y el específico del módulo; así, se llamarán "desviación hidráulica", "desviación silícica" y "desviación oxídica", a las desviaciones al standard de cal, módulo silícico, módulo de fundentes, respectivamente.

Estas desviaciones se calculan para cada una de las materias primas que intervienen en el crudo; y la suma de cada una de las desviaciones específicas multiplicada por los porcentajes en que intervenga cada materia prima se iguala a cero. De esta forma, cada módulo impuesto da lugar a una ecuación del sistema. 
Dosificaciones calculadas a base del módulo de cal

El empleo del módulo de cal para calcular la dosificación de un crudo presenta las ventajas siguientes:

a) se puede exigir un determinado contenido de silicatos y varios módulos al clínker que se desea;

b) el hecho de fijar el contenido de silicatos con el módulo de cal es tanto como determinar el tanto por ciento de $\mathrm{SiO}_{2} \mathrm{y}$, por consiguiente, el módulo silícico;

c) en el módulo de cal intervienen el mismo número de óxidos mayores que en el índice de saturación en cal, e incluso afectados de los mismos coeficientes, lo que implica la misma garantía de seguridad y validez.

Conforme se ha explicado al tratar del indice de saturación de cal, la práctica aconseja cambiar los coeficientes de la alúmina y el óxido férrico por los establecidos estadísticamente por Kühl en la expresión del standard de cal, lo cual, por analogía, debe efectuarse en el módulo de cal; se tendria, de este modo, un nuevo módulo, que por similitud podría denominarse módulo de cal standard:

$$
\mathrm{MCS}=\frac{\mathrm{CaO}-\left(1,1 \mathrm{Al}_{2} \mathrm{O}_{3}+0,7 \mathrm{Fe}_{2} \mathrm{O}_{3}\right)}{\mathrm{SiU}_{2}} .
$$

Su significado es la relación entre la cal en forma de silicatos y la sílice que con ella se combina, en la práctica.

Por consiguiente, el módulo de cal standard es al módulo de cal, lo que el standard de cal es respecto al índice de saturación de cãl.

\section{Cálculo de las desviaciones}

Un crudo de clínker de cemento portland cuyo módulo de silicatos, MS, sea $s$, deberá satisfacer:

$$
\frac{\mathrm{S}}{\mathrm{A}+\overline{\mathrm{F}}}=s
$$

y por tanto:

$$
\mathrm{S}-(\mathrm{A}+\mathrm{F}) s=\mathbf{0} .
$$

Si una materia prima cumple con la expresión anterior, es que tiene el mismo módulo de silicatos que el deseado; en caso contrario, al valor distinto de cero se le denomina desviación silícica $\left(\mathrm{D}_{\mathrm{s}}\right)$.

Un crudo cuyo módulo de fundentes (MF) sea $f$, deberá satisfacer:

$$
\frac{A}{i}=f
$$

y por lo tanto:

$$
\mathrm{A}-\mathrm{F} \cdot f=\mathbf{0} \text {. }
$$

Si una materia prima cumple con la expresión anterior, es que tiene el mismo módulo de fundentes que el deseado; en caso contrario, el valor distinto de cero recibe el nombre de desviación oxídica $\left(\mathrm{D}_{0}\right)$.

Un crudo de clínker de cemento portland cuyo módulo standard de cal, MSC, sea $\mathrm{K}$, deberá satisfacer:

$$
\mathrm{K}=\frac{\mathrm{C}-(1,1 \mathrm{~A}+\mathbf{0 , 7} \mathrm{F})}{\mathrm{S}}
$$


y por lo tanto:

$$
\mathrm{K} \cdot \mathrm{S}-\mathrm{C}+1,1 \mathrm{~A}+0,7 \mathrm{~F}=0 .
$$

Si una materia cumple con la expresión anterior es que tiene el mismo módulo standard de cal que el deseado; en caso contrario, al valor distinto de cero se le denomina "desviación cálcica" $\left(\mathrm{D}_{\mathrm{c}}\right)$.

Estas desviaciones se determinan para cada una de las materias primas que intervienen en la dosificación de crudos. Si es binaria, sólo entra en juego $\mathrm{D}_{\mathrm{c}}$; si es ternaria, intervienen $D_{c}$ y $D_{s}$; si es cuaternaria, $D_{c}, D_{s} \mathrm{y}_{0}$.

En el caso de efectuar la dosificación de crudos fijando los contenidos de $\mathrm{SC}_{3}$ y $\mathrm{SC}_{2}$, se establecen las dos ecuaciones que relacionan a ambos silicatos con el módulo standard de cal $(\mathrm{M})$ :

$$
\begin{aligned}
& \mathrm{SC}_{3}^{\prime} \%=(4,07 \mathrm{M}-7,61) \mathrm{S} \\
& \mathrm{SC}_{2} \%=(8,61-3,07 \mathrm{M}) \mathrm{S}
\end{aligned}
$$

Dividiendo miembro a miembro:

$$
\frac{\mathrm{SC}_{3} \%}{\mathrm{SC}_{2} \%}=\frac{4,07 \mathrm{M}-7,61}{8,61-3,07 \mathrm{M}}
$$

expresión de la que se deduce el valor del módulo standard de cal (M).

Es preciso hacer constar que el mismo módulo standard de cal M tendrán todos los clínkeres cuya relación $\frac{\mathrm{SC}_{2} \%}{\mathrm{SC}_{2} \%}$ coincida con la establecida y que, por consiguiente, habrá que determinar el contenido de $\mathrm{SiO}_{2}$ mediante la expresión:

$$
\mathrm{S}=\frac{\mathrm{SC}_{3} \%}{4,07 \mathrm{M}-7,61}
$$

deducida de $[\mathbf{1}]$.

El contenido de silicato suele estar comprendido entre 72 y un $80 \%$.

Al operar de la forma que se indica una ecuación establecerá que la suma de los porcentajes de las materias primas es igual a 100. La segunda ecuación indicará que la suma de las desviaciones cálcicas multiplicadas por los porcentajes de sus correspondientes materias primas es igual a cero.

La tercera ecuación se obtiene en función del contenido de $\mathrm{SiO}_{2}$ que ha de tener el crudo y su deducción es la siguiente:

Si se supone un valor para el óxido silícico, $\mathrm{SiO}_{2}$, en el clínker de $\mathrm{K}_{\mathrm{SiO}_{2}}$ y que la suma de los porcentajes de los cuatro componentes mayores de una materia prima es Am. Dicha materia prima, de contener un $\mathrm{K}_{\mathrm{SiO}_{2}}$, aportaría a la mezcla que se dosifica $\mathrm{K}_{\mathrm{SiO}_{2}} \cdot \frac{\mathrm{Am}}{100}-$; pero como únicamente contienen Sa de $\mathrm{SiO}_{2} \%$ tendrá una desviación :

$$
\mathrm{K}_{\mathbf{S 1 O}_{2}} \cdot \frac{\mathbf{A m}}{100}-\mathrm{Sa},
$$


haciendo $\frac{\mathrm{Am}}{\mathrm{i} 00}=\Sigma \mathrm{m}$ y llamando a esta desviación “desviación en sílice" $\mathrm{D}_{\mathrm{SiO}_{2}}$ :

$$
\mathrm{D}_{\mathrm{SiO}_{2}}=\left(\mathrm{K}_{\mathrm{SiO}_{2}} \cdot \Sigma \mathrm{m}-\mathrm{Sa}\right) .
$$

La tercera ecuación expresará que la suma de cada una de las desvỉaciones en sílice multiplicada por los porcentajes de sus correspondientes materias primas es igual a cero.

Conforme puede verse, la dosificación que nos ocupa ha de ser ternaria, puesto que se establecen tres ecuaciones.

\section{Sistema de ecuaciones}

Para obtener un crudo de cemento con un módulo standard de cal de $\mathrm{K}$ y un contenido de $\mathrm{SiO}_{2}$ de $\mathrm{S}_{\mathrm{k}}$, a partir de una caliza, c, una marga, m, y una arena a, constará de una parte de la primera, $x$ partes de la segunda e $y$ partes de la tercera, en peso, siendo $x$ e $y$ las cantidades que hay que determinar. La composición del crudo se deduce de las ecuaciones:

$$
\begin{aligned}
& \mathrm{D}_{\mathrm{c}_{\mathrm{c}}}+x \mathrm{D}_{\mathrm{cm}_{\mathrm{m}}}+\mathrm{D}_{\mathrm{c}_{\mathrm{a}}}=0 \\
& \mathrm{D}_{\mathrm{SiO}_{2}}+x \mathrm{D}_{\mathrm{SiO}_{2} \mathrm{~m}}+y \mathrm{D}_{\mathrm{SiO}_{\mathrm{a}}}=0 .
\end{aligned}
$$

Resuelto el sistema por cualquiera de los procedimientos habituales, resulta:

$$
\begin{aligned}
& \mathrm{X}=\frac{\mathrm{D}_{\mathrm{c}_{\mathrm{a}}} \cdot \mathrm{D}_{\mathrm{SiO}_{\mathrm{c}_{\mathrm{c}}}}-\mathrm{D}_{\mathrm{c}_{\mathrm{c}}} \cdot \mathrm{D}_{\mathrm{SiO}_{\mathrm{a}}}}{\mathrm{D}_{\mathrm{c}_{\mathrm{m}}} \cdot \mathrm{D}_{\mathrm{SiO}_{\mathrm{a}_{\mathrm{a}}}}-\mathrm{D}_{\mathrm{c}_{\mathrm{a}}} \cdot \mathrm{D}_{\mathrm{SiO}_{2} \mathrm{~m}}} \\
& y=\frac{\mathrm{D}_{\mathrm{c}_{\mathrm{c}}} \cdot \mathrm{D}_{\mathrm{SiO}_{2}}-\mathrm{D}_{\mathrm{c}_{\mathrm{m}}} \cdot \mathrm{D}_{\mathrm{SiO}_{\mathrm{c}_{\mathrm{c}}}}}{\mathrm{D}_{\mathrm{c}_{\mathrm{m}}} \cdot \mathrm{D}_{\mathrm{SiO}_{2^{2}}{ }_{\mathrm{a}}}^{\cdot}-\mathrm{D}_{\mathrm{c}_{\mathrm{a}}} \cdot \mathrm{D}_{\mathrm{SiO}_{\mathrm{m}_{\mathrm{m}}}}} .
\end{aligned}
$$

Las desviaciones han de calcularse con cuatro cifras decimales, afectadas de su signo correspondiente. A veces resulta en los cálculos alguna cantidad negativa para la proporción de alguno de los componentes, y ello indica que, con los materiales disponibles no se puede dosificar un crudo que cumpla simultáneamente las condiciones impuestas.

Las expresiones que indican los porcentajes en que han de mezclarse los materiales, son:

$$
\begin{aligned}
& \text { Caliza } \%=\frac{100}{1+\frac{x+y}{y}}, \\
& \text { Marga } \%=\frac{100}{1+\frac{x+y}{}}, \\
& \text { Arena } \%=\frac{100}{1+x+y} .
\end{aligned}
$$




\section{EJEMPLO 1}

Con las siguientes materias primas:

\begin{tabular}{|c|c|c|c|c|c|c|c|c|c|c|c|}
\hline Caliza & & $\%$ & & Marga & & $\%$ & & Arena & & $\%$ & \\
\hline $\mathrm{SiO}_{2}$ & $=$ & 5,0 & & $\mathrm{SiO}_{2}$ & $=$ & 32,0 & & $\mathrm{SiO}_{2}$ & $=$ & 87,0 & \\
\hline $\mathrm{Al}_{2} \mathrm{O}_{3}$ & $=$ & 2,0 & & $\mathrm{Al}_{2} \mathrm{O}_{3}$ & $=$ & 12,0 & & $\mathrm{Al}_{2} \mathrm{O}_{3}$ & $=$ & 2,0 & \\
\hline $\mathrm{Fe}_{2} \mathrm{O}_{3}$ & $=$ & 1,0 & & $\mathrm{Fe}_{2} \mathrm{O}_{3}$ & $=$ & 5,0 & & $\mathrm{Fe}_{2} \mathrm{O}_{3}$ & $=$ & 5,0 & \\
\hline $\mathrm{CaO}$ & $=$ & 50,0 & & $\mathrm{CaO}$ & $=$ & 25,0 & & $\mathrm{CaO}$ & $=$ & 3,0 & \\
\hline $\mathrm{MgO}$ & $=$ & 2,0 & $60 \%$ & $\mathrm{MgO}$ & $=$ & 3,4 & $77,4 \%$ & $\mathrm{MgO}$ & $=$ & 0,5 & $97,5 \%$ \\
\hline Alc & $=$ & 0,8 & & Alc & $=$ & 3,0 & & Alc & $=$ & 0,5 & \\
\hline $\mathrm{PF}$ & $=$ & 39,2 & & $\mathrm{PF}$ & $=$ & 19,6 & & PF & $=$ & 2,0 & \\
\hline & & 100,0 & & & & 100,0 & & & & 100,0 & $\%$ \\
\hline
\end{tabular}

hallar la dosificación para obtener un clínker con un $60 \%$ de $\mathrm{SC}_{3}$ y un $15 \%$ de $\mathrm{SC}_{2}$.

Cálculo del Módulo standard de cal

$$
\begin{aligned}
& 60=(4,07 \mathrm{M}-7,61) \mathrm{S}, \\
& 15=(8,61-3,07 \mathrm{M}) \mathrm{S},
\end{aligned}
$$

y dividiendo miembro a miembro:

$$
4=-\frac{4,07 \mathrm{M}-7,61}{8,51-3,67 \mathrm{M}}, \text { de donde } \mathrm{M}=2,57 \text {; }
$$

ahora bien, el mismo valor 2,57 tendrán todos los clínkeres cuya relación $\frac{\mathrm{SC}_{3} \%}{\mathrm{SC}_{2} \%}$ sea 4. Por consiguiente, habrá que determinar el contenido de $\mathrm{SiO}_{2}$ mediante la ecuación:

$$
\mathrm{SiO}_{2} \%=\frac{60}{4,07 \times 2,57-7,61} \quad \mathrm{SiO}_{2}=21,5 \%
$$

\section{Cálculo de las desviaciones}

Desviación cálcica de la caliza $\left(\mathrm{D}_{\mathrm{c}_{\mathrm{c}}}\right)$ :

$$
2,57 \times 5,0+1,1 \times 2+0,7 \times 1-50,0=-34,2500 .
$$

Desviación en sílice de la caliza $\left(\mathrm{D}_{\mathrm{SiO}_{2} \mathrm{c}}\right)$ :

$$
21,05 \times 0,50-5,0=7,6300 .
$$

Desviación cálcica de la marga $\left(\mathrm{D}_{\mathrm{c}_{\mathrm{m}}}\right)$ :

$$
2,57 \times 32,0+1,1 \times 12,0+0,7 \times 5,0-25,0=75,3400 .
$$

Desviación en sílice de la marga $\left(\mathrm{D}_{\mathrm{Si}_{2} \mathrm{~m}}\right)$ :

$$
21,05 \times 0,774-32=-15,7073 .
$$


Desviación cálcica de la arena $\left(\mathrm{D}_{\mathrm{c}_{\mathrm{a}}}\right)$ :

$$
2,57 \times 87,0+1,1 \times 2+0,7 \times 5-3,0=226,2900 .
$$

Desviación en sílice de la arena $\left(\mathrm{D}_{\mathrm{Si}_{2 \mathrm{a}}^{\mathrm{a}}}\right)$ :

$$
21,05 \times 0,975-87=-66,4763 .
$$

Por consiguiente, resumiendo:

$$
\begin{aligned}
& \text { Caliza }\left\{\begin{array}{l}
\text { Desviación cálcica }=-34,2500 . \\
\text { Desviación en sílice }= \\
7,6300 .
\end{array}\right. \\
& \text { Marga }\left\{\begin{array}{l}
\text { Desviación en sílice }=-15,7073 . \\
\text { Desviación cálcica }=75,3400 .
\end{array}\right. \\
& \text { Arena }\left\{\begin{array}{l}
\text { Desviación cálcica }=226,2900 . \\
\text { Desviación en sílice }=-66,4763 .
\end{array}\right.
\end{aligned}
$$

Planteamiento de las ecuaciones:

$$
\begin{gathered}
-34,2500+75,3400 x+226,2900 y=0 \\
7,6300-15,7073 x-66,4763 y=0 \\
\Delta x=\left|\begin{array}{cc}
34,25 & 226,29 \\
7,63 & -66,4763
\end{array}\right|=-2.276,8133+1.726,5972=-550,2206 . \\
\Delta y=\left|\begin{array}{rr}
75,34 & 34,25 \\
15,7073 & 7,63
\end{array}\right|=-574,8442+537,9750=-36,8692 . \\
\Delta=\left|\begin{array}{lr}
75,34 \\
15,7073 & -626,29
\end{array}\right|=-5.008,3244+3.554,4049=-1.453 .9195 . \\
x=\frac{\Delta x}{\Delta}=\frac{-550,2206}{-1.453,9195}=0,3784 \\
y=\frac{\Delta y}{\Delta}=\frac{-36,8692}{-1.453,9195}=0,0254 .
\end{gathered}
$$

La dosificación será :

1 parte de caliza,

0,3784 partes de marga,

0,0254 partes de arena, 
que en tanto por ciento será:

$$
\begin{aligned}
& \text { Caliza }= \frac{\%}{71,23} \\
& \text { Marga }= 26,96 \\
& \text { Arena }=\frac{1,81}{100,00 \% .}
\end{aligned}
$$

La composición del crudo será:

$$
\begin{aligned}
& \text { Caliza: } \mathrm{SiO}_{2}=0,7123 \times 5,0=3,5615 \\
& \mathrm{Al}_{2} \mathrm{O}_{3}=0,7123 \times 2,0=1,4246 \\
& \mathrm{Fe}_{2} \mathrm{O}_{3}=0,7123 \times 1,0=0,7123 \\
& \mathrm{CaO}=0,7123 \times 50,0=35,6150 \\
& \mathrm{MgO}=0,7123 \times 2,0=1,4246 \\
& \text { Marga: } \mathrm{SiO}_{2}=0,2696 \times 32,0=8,6272 \\
& \mathrm{Al}_{2} \mathrm{O}_{3}=0,2696 \times 12,0=3,2352 \\
& \mathrm{Fe}_{2} \mathrm{O}_{3}=0,2696 \times 5,0=1,3480 \\
& \mathrm{CaO}=0,2696 \times 25,0=6,7400 \\
& \mathrm{MgO}=0,2696 \times 3,4=0,9166 \\
& \text { Arena: } \mathrm{SiO}_{2}=0,0181 \times 87,0=1,5747 \\
& \mathrm{Al}_{2} \mathrm{O}_{3}=0,0181 \times 2,0=0,0362 \\
& \mathrm{Fe}_{2} \mathrm{O}_{3}=0,0181 \times 5,0=0,0905 \\
& \mathrm{CaO}=0,0181 \times 3,0=0,0543 \\
& \mathrm{MgO}=0,0181 \times 0,5=0,0091 .
\end{aligned}
$$

Y por consiguiente:

$$
\begin{aligned}
& \frac{\%}{13,76} \\
\mathrm{SiO}_{2}= & 4,70 \\
\mathrm{Al}_{2} \mathrm{O}_{3} & =2,15 \\
\mathrm{Fe}_{2} \mathrm{O}_{3} & =42,41 \\
\mathrm{CaO} & =2,3 \overline{65,37 \%} \\
\mathrm{MgO} & =\underline{34,63} \\
\text { Materias } & \\
\text { volátiles... } &
\end{aligned}
$$


El clínker obtenido será:

$$
\begin{aligned}
& \% \\
& \mathrm{SiO}_{2}=21,05 \\
& \mathrm{Al}_{2} \mathrm{O}_{3}=7,19 \\
& \mathrm{Fe}_{2} \mathrm{O}_{3}=3,29 \\
& \mathrm{CaO}=64,88 \\
& \mathrm{MgO}=3,59 \\
& 100,00 \% \text {. }
\end{aligned}
$$

\section{COMPROBACION :}

Módulo standard de cal:

$$
\begin{aligned}
& \frac{64,88-(1,1 \times 7,19+0,7 \times 3,29)}{21,05}=2,59 \\
& \% \\
& \mathrm{SiO}_{2}=21,05 \\
& \mathrm{SC}_{3}=61,70 \\
& \mathrm{SC}_{2}=13,90 \quad 75,60 \% \text { silicatos. }
\end{aligned}
$$

\section{procedimiento gráfico de dosthcación de crudos}

Un procedimiento rápido para calcular si las materias primas de que se dispone satisfacen las condiciones impuestas a un crudo determinado es el método gráfico debido a Fenarolli (8), que incluso permite obtener una valoración cuantitativa muy aceptable.

En dicho sistema gráfico es posible representar la mezcla del crudo (integrada por los cuatro óxidos fundamentales) por un punto del espacio, correspondiente al interior de un tetraedro (fig. 1). Las aristas CS y AF son, respectivamente, perpendiculares a las caras SAF y SFC. En la citada figura se ha representado la mezcla de un crudo $M$, cuyas proyecciones sobre las caras $S A F$ y $S F C$ son $M_{1}$ y $M_{2}$.

Si al tetraedro de la figura 1 se proyecta sobre dos planos paralelos a las caras SAF y SFC y ambas proyecciones se disponen en un plano único, se obtendría una representación análoga a la figura 2. 
Cada vértice del tetraedro primitivo de la figura 1 se ha designado con una letra correspondiente a un óxido fundamental del clínker, de acuerdo con el convenio aceptado internacionalmente $\left(\mathrm{C}=\mathrm{CaO} ; \mathrm{A}=\mathrm{Al}_{2} \mathrm{O}_{3} ; \mathrm{F}=\mathrm{F}_{2} \mathrm{O}_{3} ; \mathrm{S}=\mathrm{SiO}_{2}\right)$.

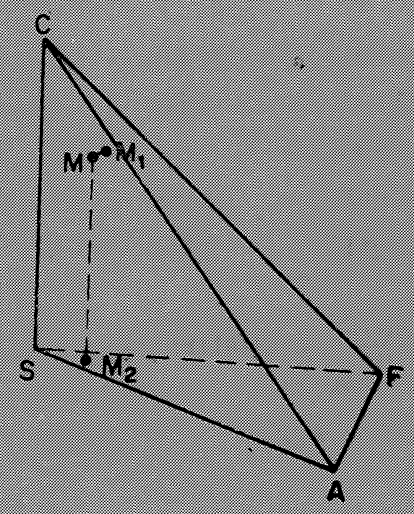

Fig. 1

Mediante la representación de la figura 2 ha sido posible disponer en un plano el sistema espacial primitivo. Dicha representación está constituida por dos triángulos; con ellos, el 1, se puede representar gráficamente el valor de la relación $\frac{\mathrm{C}}{\mathrm{S}+\mathrm{R}_{2} \mathrm{O}_{3}}$ (módulo hidráulico), y la relación $\frac{\mathrm{S}}{\mathrm{R}_{2} \mathrm{O}_{3}}$ (módulo silícico); mientras que el triángulo número 2 permite representar gráficamente el módulo de fundentes $\frac{\mathrm{A}}{\mathrm{F}}$.

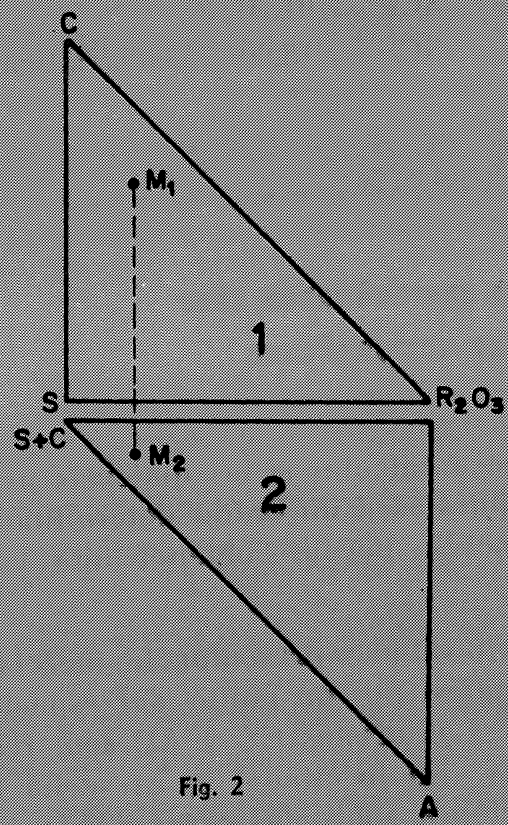

En el triángulo número 1 , de la figura 2 , las rectas paralelas al lado $\mathrm{S}-\mathrm{R}_{2} \mathrm{O}_{3}$ constituyen el lugar geométrico de las composiciones que tienen igual módulo hidráulico. En el citado triángulo toda recta que parta del vértice $\mathrm{C}$ y se dirija al lado $\mathrm{S}-\mathrm{R}_{2} \mathrm{O}_{3}$ 
es el lugar geométrico de los puntos que gozan del mismo módulo silícico. En el triángulo 2, de la figura 2, la recta que parte del vértice $\mathrm{S}+\mathrm{C}$ y se dirige al lado $\mathrm{AF}$ representa el conjunto de los puntos que tienen el mismo módulo de fundentes.

Es obvio que por medio de este sistema gráfico se pueden representar la mayor parte de los compuestos cuaternarios (en el caso presente, $\mathrm{CaO}-\mathrm{SiO}_{2}-\mathrm{Al}_{2} \mathrm{O}_{3}-\mathrm{Fe}_{2} \mathrm{O}_{3}$ ), en función de los tres módulos característicos.

Para mayor comodidad de representación se han dispuesto los triángulos 1 y 2 conforme se indica en la figura 3 , de manera que tengan común las hipotenusas. Esta traslación geométrica no altera el concepto en absoluto, sino que tiene la ventaja de hacer más fácil la interpretación del sistema.

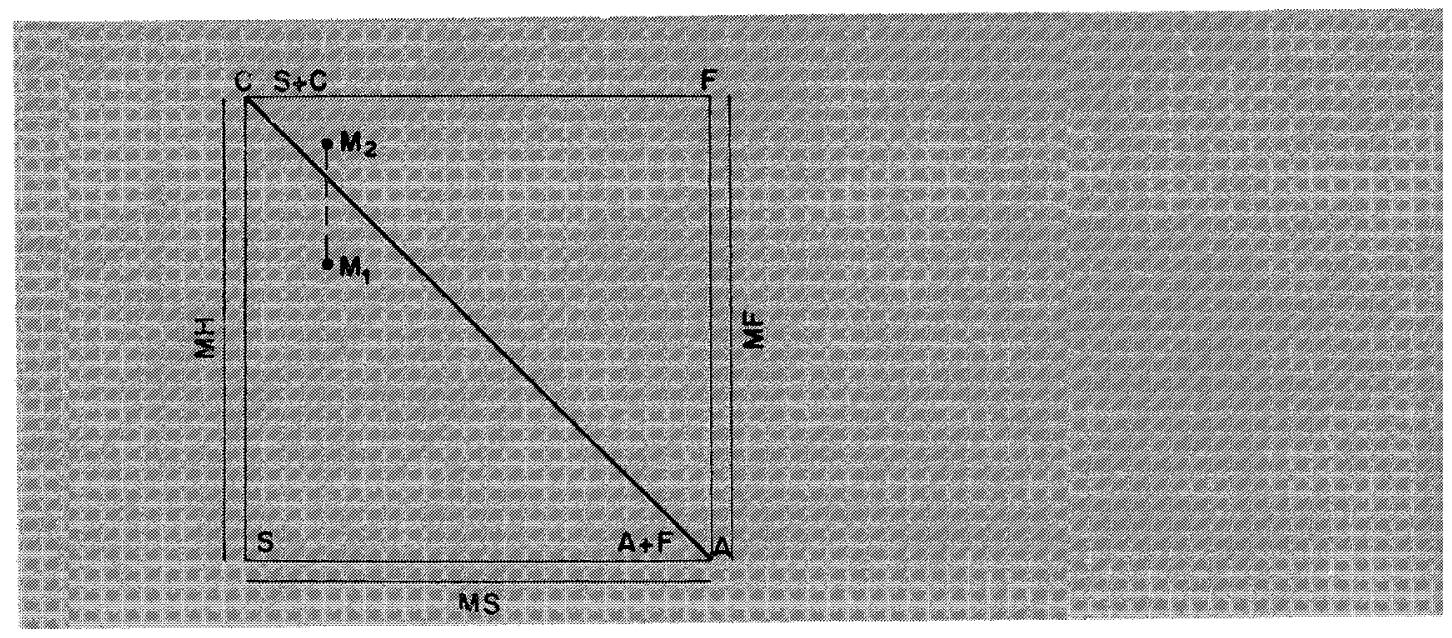

Fig. 3

En el triángulo superior $(\mathrm{S}+\mathrm{C}) \mathrm{AF}$, que en esencia representa el diagrama de fundentes, se fijan los puntos representativos del $\mathrm{AC}_{9}, \mathrm{FAC}_{4} \mathrm{y} \mathrm{FC}_{2}$ (fig. 4).

Sobre el lado $(S+C) F$ se disponen los porcentajes de los fundentes de aquellas composiciones cuyo módulo de fundentes esté comprendido entre 0 y 0,64 . Sobre la hipotenusa $(S+C)$ A se dispone el tanto por ciento de los fundentes, cuyo módulo varia entre 0,64 e infinito.

Sobre el lado $\mathrm{S}$ C del triángulo inferior $\mathrm{CSR}_{2} \mathrm{O}_{3}$ se encuentran los puntos de partida de las rectas de igual módulo de cal o igual módulo de standard de cal, las cuales deberán trazarse en función del valor del módulo de fundentes propio de la composición que se estudia. La escala de los valores del módulo de fundentes utilizada para trazar esta recta se lleva sobre la hipotenusa, a partir de su encuentro con la recta $\mathrm{FA}$ del triángulo $(\mathrm{S}+\mathrm{C}$ ) $\mathrm{AF}$, partiendo de los puntos que forman las especies $\mathrm{AC}_{3}, \mathrm{FAC}_{4}$ y $\mathrm{FC}_{2}$.

Sobre el lado $\mathrm{S}$ C se sitúan los puntos representativos de las diferentes relaciones entre la cal combinada con la sílice y ésta, es decir, el módulo de cal y el módulo de standard de cal, las cuales estarán comprendidas entre 1,87 y 2,80. Para un valor fijo del módulo de fundentes, por ejemplo, 2,50, se pueden trazar las rectas con módulo de cal de 2,80 y 1,87 . De este modo se obtiene un triángulo que comprende todas las composiciones cuyo módulo de fundentes sea 2,50 y su módulo de cal o módulo standard de cal variable entre 1,87 y 2,80 (fig. 4). 
Variando el valor del módulo de fundentes se puede construir, de forma análoga, una serie completa de triángulos $\mathrm{SC}_{3}-\mathrm{SC}_{2}$-fundentes, que delimitarán teóricamente las posibles composiciones con módulo de fundentes entre cero e infinito.

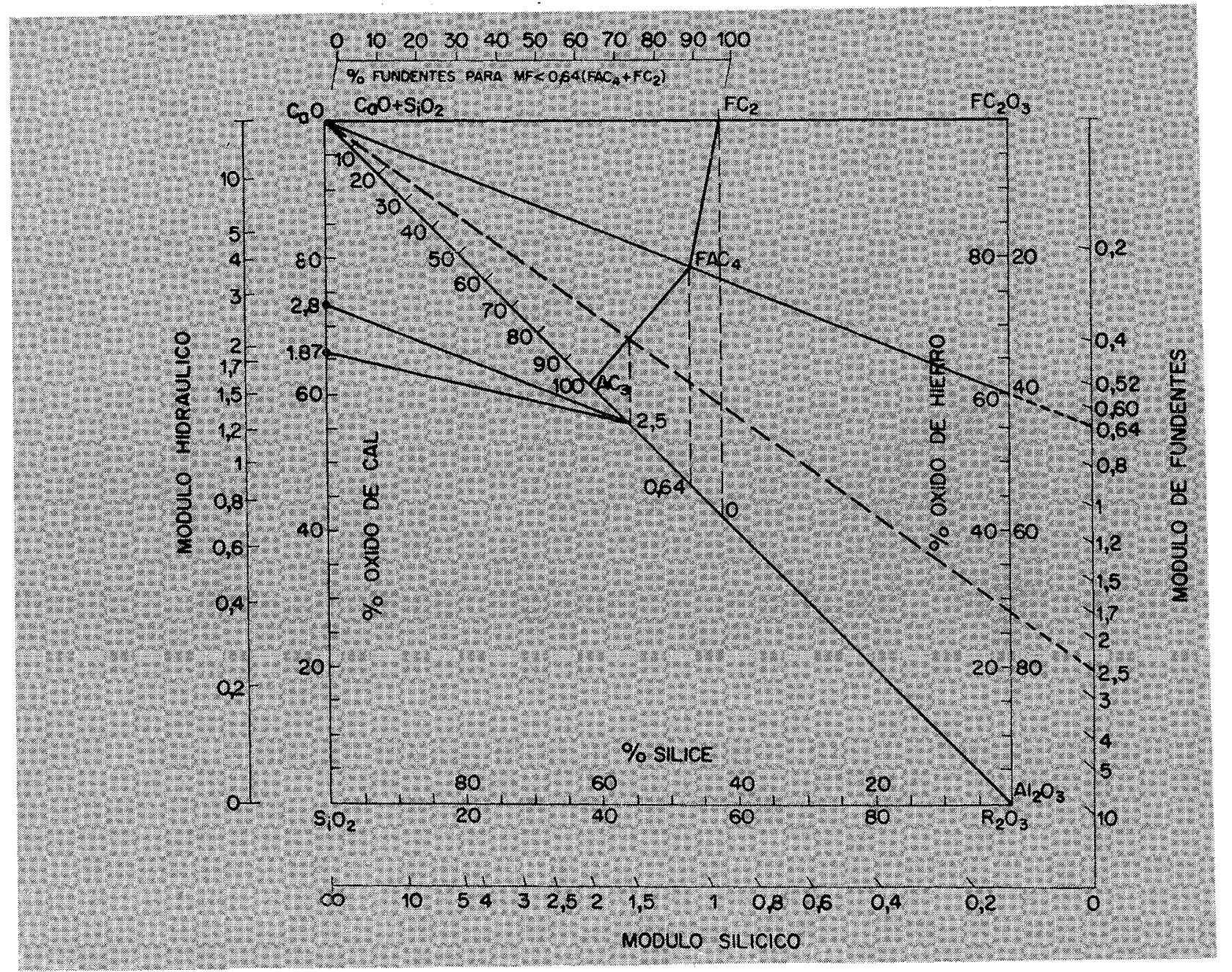

Fig. 4

Los cálculos previos para evaluar una mezcla de un crudo se pueden efectuar con suficiente exactitud, disponiendo el diagrama de la figura 4 en un papel milimetrado de absoluta garantia, de forma que el lado del cuadrado del sistema representado tenga un total de $50 \mathrm{~cm}$. Dicha representación conviene realizarla sobre papel milimetrado vegetal con el fin de su más fácil reproducción.

Mediante este sistema de representación se puede expresar la composición del crudo en función de los tres módulos fundamentales: módulo de cal, módulo del standard de cal, módulo silícico y módulo de fundentes.

Como la representación se efectúa sobre un plano, únicamente pueden adoptarse dos variables independientes, y aceptar como fijo de los tres módulos, expresando la composición en función de los otros dos. 
El módulo que debe adoptarse como fijo es siempre el módulo de fundentes; ya que si en el gráfico se trazan las líneas correspondientes a los módulos de cal extremos para los módulos de fundentes 1 y 2,7, se observa que el área que representa las composiciones totales de los cementos portland varía muy poco al alterar el valor del módulo de fundentes.

Por consiguiente, las materias primas y el crudo se pueden representar en función del módulo del standard de cal y del módulo silícico; este último puede sustituirse por un valor determinado de $\mathrm{SiO}_{2} \%$ en función de unos contenidos de $\mathrm{SC}_{3}$ y $\mathrm{SC}_{2}$ deseados, cuya deducción se ha realizado al exponer el procedimiento algebraico.

El técnico, basado en esta representación, puede predecir, con suficiente rapidez, si con las materias primas disponibles es posible obtener un clínker de cemento portland determinado:

\section{Ejemplo}

Con las materias primas del ejemplo 1 buscar, gráficamente, la dosificación de un crudo para hallar un clínker con un $60 \%$ de $\mathrm{SC}_{3}$ y un $15 \%$ de $\mathrm{SC}_{2}$ :

\section{Resolución}

En primer lugar hay que reducir los análisis químicos de las materias primas, únicamente a los cuatro óxidos fundamentales para que puedan ser representados en la gráfica:

\begin{tabular}{|c|c|c|c|c|}
\hline & & Caliza & Marga & Arena \\
\hline $\mathrm{SiO}_{2}$ & $=$ & 8,6 & 43,2 & 89,7 \\
\hline $\mathrm{Al}_{2} \mathrm{O}_{3}$ & $=$ & 3,4 & 16,2 & 2,0 \\
\hline $\mathrm{Fe}_{2} \mathrm{O}_{3}$ & $=$ & 1,7 & 6,8 & 5,2 \\
\hline $\mathrm{CaO}$ & $=$ & 86,3 & 33,8 & 3,1 \\
\hline & & 100,0 & 100,0 & 100,0 \\
\hline
\end{tabular}

En la figura 5 la caliza está representada por el punto C, la marga por M y la arena por A.

Efectuando el cálculo del módulo standard de cal y el contenido de $\mathrm{SiO}_{2}$ conforme se ha expuesto en el método algebraico,

$$
\begin{aligned}
& \text { Módulo del standard de cal }=2,57, \\
& \mathrm{SiO}_{2} \% \\
& =21,05,
\end{aligned}
$$

el módulo de fundentes se mantiene constante e igual a 2,5.

De esta forma el punto representativo de la mezcla deseada será el $\mathbf{X}$.

Si se une el punto $\mathrm{C}$ con el $\mathrm{M}$, la recta que los une no pasa por el punto $\mathrm{X}$, lo que indica que con la caliza y la marga no se puede obtener la mezcla deseada. 
Se une el punto $\mathrm{C}$ con $\mathrm{X}$, y el $\mathrm{M}$ con el $\mathrm{A}$, ambos segmentos se cortan en el punto $\mathrm{S}$, que nos indica la proporción de cada uno de los tres materiales que intervienen en la dosificación:

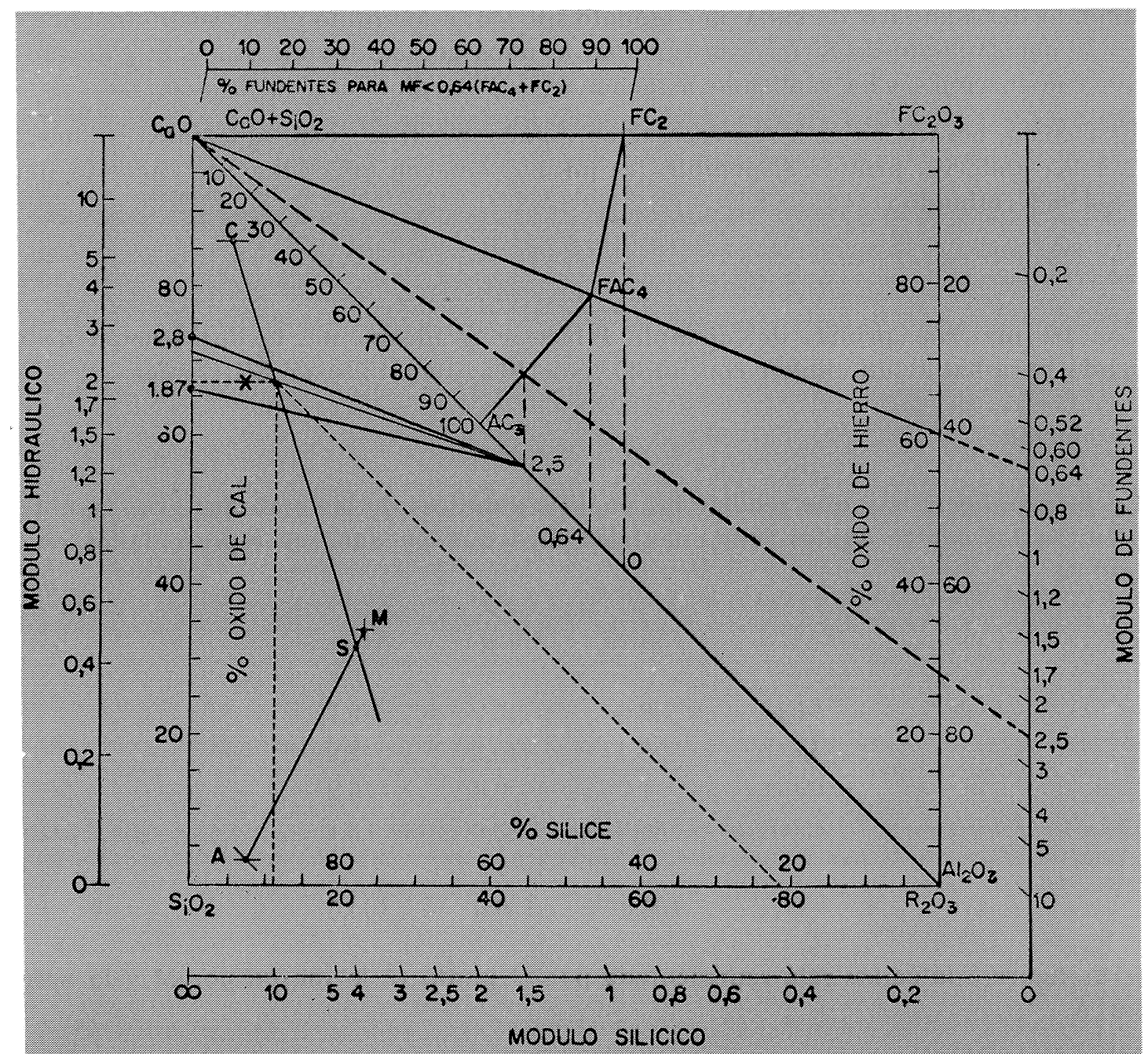

Fig. 5

$$
\frac{\text { caliza }}{\text { mezclas }}=\frac{\overline{X S}}{\mathrm{XC}} \frac{18,10}{9,8}
$$

La mezcla binaria S está compuesta:

$$
\text { Caliza } \%=\frac{\frac{\text { marga }}{\text { arena }}=\frac{\text { A S }}{\mathrm{S} . \mathrm{M}}-17,05}{0,2}
$$




$$
\begin{aligned}
& \text { Marga } \%=\frac{\overline{\mathrm{AS}} \cdot 100}{\overline{\mathrm{AM}}} \times 0,35=31,6 \% . \\
& \text { Arena } \%=\frac{\overline{\mathrm{SM}} \cdot 100}{\mathrm{~A} \mathrm{M}} \times 0,35=0,4 \% .
\end{aligned}
$$

La composición ternaria será:

$$
\begin{aligned}
& \text { Caliza }= \frac{\%}{65} \\
& \text { Marga }= \mathbf{3 4 , 6} \\
& \text { Arena }= 0,4 \\
& \hline 100,0 \%
\end{aligned}
$$

La composición del clínker serả:

$$
\begin{aligned}
& \text { Caliza }= 8,6 \times 0,65=5,59 \\
& 3,4 \times 0,65= 2,21 \\
& 1,7 \times 0,65= 1,11 \\
& 86,3 \times 0,65=56,09 \\
& \text { Marga }= 432 \times 0,346=14,95 \\
& 16,2 \times 0,316=5,61 \\
& 6,8 \times 0,346=2,35 \\
& 33,8 \times 0,346=11,69 \\
& \text { Arena }= 89,7 \times 0,004=0,36 \\
& 2,0 \times 0,004=0,008 \\
& 5,2 \times 0,004=0,020 \\
& 3,1 \times 0,004=0,012 \\
& \mathrm{SiO}_{2}=20,90 \\
& \mathrm{Al}_{2} \mathrm{O}_{3}=7,83 \\
& \mathrm{Fe}_{2} \mathrm{O}_{3}=3,48 \\
& \mathrm{CaO}^{2}=67,79
\end{aligned}
$$

Valores éstos que corresponden con los dados por el diagrama.

\section{COMPROBACION :}

$$
\text { Módulo de cal }=\frac{67,79-(1,65 \times 7,8+0,35 \times 3,5)}{20,9}=2,57 .
$$

\section{Gibliografía}

(1) L. Santarelli: Elementi di chimica.

(2) W. A. KInd: Sonderzemente. Leningrad 1932.

(3) W. A. KIND: Chemische Kennzeichnung des Portland-Zementes.

(4) W. A. King: Zement, núm. 8, pág. 19-24 (1935) Leningrad 1932.

(5) W. A. Kınd: Zement, núm. 6, pág. 26 (1936).

(6) S. D. Okonokow: Die Berechnung der Portlandzement-Rohmischung. 1947.

(7) J. Calleja: Cemento portland, cảlculo e interpretación de datos químicos. Instituto "Eduardo Torroja" de la Construcción y del Cemento. Madrid 1960.

(8) Fenarolli: Il Cemento, pág. 85, 1929. 